\title{
Tobacco treatment TrAining Network in Crete (TiTAN-Crete): protocol for a controlled before-after study
}

\author{
Charis Girvalaki ${ }^{1}$, Sophio Papadakis ${ }^{1,2,3}$, Constontine Vardavas ${ }^{1}$, Andrew Pipe ${ }^{2,3}$, Christos Lionis ${ }^{1}$
}

\begin{abstract}
INTRODUction Rates of tobacco use in Greece are among the highest in Europe and are responsible for an enormous burden of chronic disease and death. A large proportion of tobacco users report an interest in quitting. Family medicine practices have been identified as important settings for identifying tobacco users, delivering advice to quit smoking, and providing tobacco treatment interventions.

The 5A's (ask, advice, assess, assist, arrange) schema is an evidence-based model for addressing tobacco use in clinical settings. The rates at which primary care clinicians in Greece address tobacco use in their practice is unknown but, as in other countries, is understood to be sub-optimal. This paper describes the rationale, design, and protocol for a pre/post, controlled study to compare the effectiveness of a smoking cessation intervention delivered in primary care practices in Heraklion, Greece. The TiTAN-Crete intervention includes a 1-day tobacco treatment training program, dissemination of provider and patient resources and two booster training sessions. Participating providers and a cross-sectional sample of patients from their practices, will be surveyed. Outcome measures include changes in provider attitudes and beliefs, perceived behavioral control, intentions, and barriers related to smoking cessation treatment delivery.

Rates at which providers deliver tobacco treatment to patients will be measured by patient report of 5As delivery. Multi-level modeling will be used to examine the effects of the intervention. This study will lead to a better understanding of how to best assist clinicians in Greece to enhance the rates at which smoking cessation treatments are delivered to smokers.
\end{abstract}

TRIAL REGISTRATION: ISRCTN10306198

ABBREVIATIONS

GP - General Practitioner

OMSC - Ottawa Model for Smoking Cessation

TITAN CRETE - Tobacco treatment TrAining Network in Crete

\section{AFFILIATION \\ 1 Clinic of Social and Family Medicine, Faculty of Medicine, University of Crete, Heraklion, Crete, Greece \\ 2 Division of Prevention and Rehabilitation, University of Ottawa Heart Institute, Ottawa, Ontario, Canada \\ 3 Faculty of Medicine, University of Ottawa, \\ Ottawa, Ontario, Canada}

CORRESPONDENCE TO: Charis Girvalaki, Clinic of Social and Family Medicine, Faculty of Medicine, University of Crete, Heraklion, Crete, Greece, PO Box 2208. Email address: Charis@ tobcontrol.eu

\section{KEY WORDS:}

smoking cessation, primary care, study protocol, training, Greece

\section{INTRODUCTION}

Greece has one of the highest rates of tobacco use among members of the European Union (EU), estimated at slightly above $38 \%$ of the adult population ${ }^{1}$. In addition, high rates of tobacco addiction have been reported, with $53 \%$ of Greek tobacco users consuming more than 20 cigarettes per day, a rate that is also one of the highest in the $\mathrm{EU}^{2,3}$. Tobacco use is responsible for an enormous burden of chronic disease and death ${ }^{4}$. Moreover, tobacco use places an extraordinary burden on the national health care system and is responsible for an estimated 200,000 hospital admissions ( $8.9 \%$ of the national total), with attributable hospital treatment costs calculated at over 554 million Euro, which represents $10.7 \%$ of the national hospital budget ${ }^{5}$.
Family practice has been identified as an important setting for the delivery of smoking cessation treatment ${ }^{6,7}$. General Practitioners (GPs) working in primary care are well positioned to identify and intervene with smokers ${ }^{8}$. The $5 \mathrm{~A}^{\text {'s }}$ of smoking cessation are an internationally recognized evidence-based schema to guide interventions with tobacco users in clinical settings including primary care ${ }^{9,}{ }^{10}$. The $5 \mathrm{As}$ include: ask patients about smoking status; provide brief quit smoking advice; assess readiness to quit smoking; assist patients with making a quit attempt using behavioral techniques and pharmacotherapies; and, arrange follow-up support throughout the quitting process. The rates at which primary care clinicians in Greece address tobacco use in their practice is unknown but, as in other countries, is understood to be sub-optimal ${ }^{1,11-13}$. 
Addressing barriers to the delivery of tobacco treatment including clinician knowledge, attitudes, skills, and time constraints is necessary to increase the uptake of tobacco treatment interventions in primary care setting $5^{5,14-17}$. The 'Ottawa Model for Smoking Cessation' (OMSC), is a multicomponent intervention for addressing tobacco use with smokers in primary care settings that is based on the $5 \mathrm{~A}$ 's model $^{11,18,19}$. Evaluation of the OMSC program in Canadian primary care practices reveals a significant improvement in the rates at which evidence-based tobacco treatments are delivered in a large sample of primary care practices ${ }^{11,20}$. The extent to which this model can be used to influence the practice behaviors of primary care clinicians in countries such as Greece is unknown. The primary objective of this study, therefore, was to test the effectiveness of an adaptation of the Ottawa Model among a sample of general practitioners in Greece in increasing provider knowledge, attitudes, confidence and rates of tobacco treatment delivery. In this paper, we describe the study rationale, design, methods, and protocol.

\section{METHODS}

\section{Study Design}

A pre-post, controlled study design will be employed. The study design schema is presented in Figure 1. From each of the participating practices, a cross-sectional sample of eligible tobacco users will be recruited before and after the 'Tobacco treatment TrAining Network in Crete' (TiTANCrete) intervention program is implemented to assess changes in the tobacco treatment outcomes of interest in both intervention and control practices. This trial received approval from the University Hospital of Heraklion Ethics Board (ref\# 18078) and was registered at clinicaltrials.gov (identifier: ISRCTN10306198).

\section{Figure 1: A before after, controlled study design}

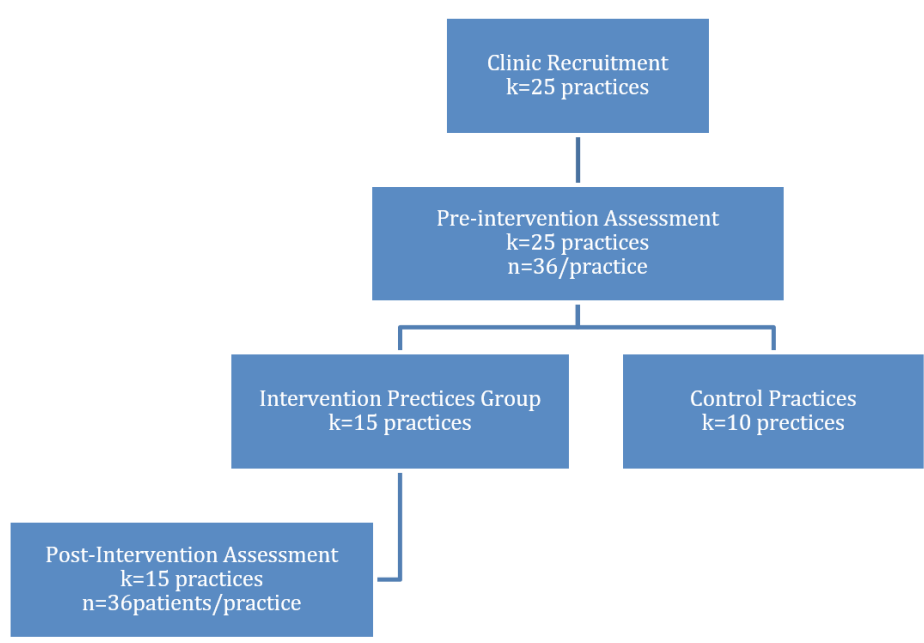

Figure 2: Conceptual model provider perspective

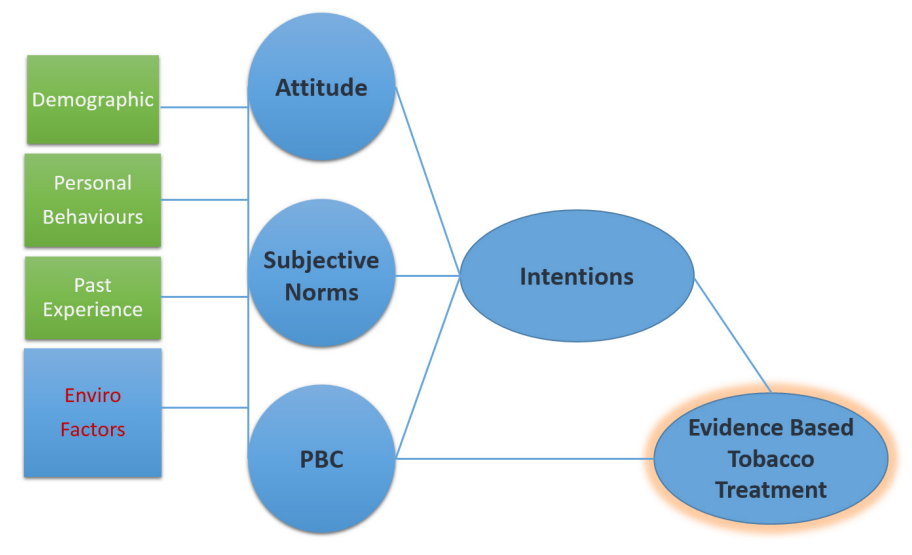

\section{Setting and participants}

The study will take place in the region of Heraklion, Crete, Greece. Primary care clinics within the Practice Based Research Network, a primary care provider group affiliated with the Clinic of Social and Family Medicine at the University of Crete will be exposed to the intervention program. A sample of control primary care practices will be recruited from the city of Rethymno (Crete, Greece); they will not be exposed to the intervention. A two-level recruitment strategy will be employed in which primary care providers will be recruited and then, from each provider's practice, a cross-sectional sample of eligible patients will be recruited before and after the intervention program.

\section{Intervention Framework}

The intervention program seeks to change behavior of both the providers and in turn their patients who smoke. Ajzen's Theory of Planned Behavior (TPB) has been used to guide intervention design ${ }^{21}$. Figure 2 provides a schematic representation of the conceptual framework for delivery of evidence-based tobacco treatments by providers. We will employ several tactics, based on the TPB, within the multi-component intervention to enhance the program's uptake into practice. Specifically the intervention program will target: i) provider attitudes towards tobacco use and treatment; ii) the establishment of new social and clinical norms related to tobacco treatment in primary care practice settings (normative beliefs); iii) increasing providers' perceptions about the ease of delivering tobacco treatment (perceived behavioral control); and, iv) providers intentions to deliver 5As treatments to patients. TPB has been applied in previous evaluations of smoking cessation interventions ${ }^{22}$.

\section{Intervention Comparators}

\section{TiT A N Crete Intervention Program}

The TiTAN Crete program has adapted the existing curricula and resources originally developed at the University of Ottawa 
Heart Institute and which are specific to primary practice setting $\mathrm{s}^{20}$. To facilitate maximum uptake the intervention program was adapted to reflect: language; cultural appropriateness; local patient beliefs and attitudes regarding tobacco-use and cessation; local social and clinical norms; provider perceptions surrounding 5As delivery; and practice characteristics. The TiTAN-Crete intervention includes a 1-day tobacco treatment training program for general practitioners, and the dissemination of provider and patient resources.

\section{ProviderTraining}

The training program consists of a one-day core session addressing tobacco use with patients in the context of a busy primary care practice setting. The training includes information regarding: the neuro-biology of nicotine addiction; health effects of tobacco use; the role of primary care providers in motivating cessation; evidence-based tobacco treatment practices; techniques for delivering brief cessation advice; the use of first-line cessation pharmacotherapy; motivational interviewing techniques; and special populations. Two booster 3-hour sessions will be delivered 1- and 3-months after the core training. The booster sessions are designed to reinforce the adoption of new practice behaviors and offer practical skills-based training focused on patients in the GP's own practice. Booster session \#1 will focus on conducting initial and follow-up smoking cessation visits for patients ready to quit. Booster session \#2 will focus on cognitive behavioral counseling techniques. The curriculum design was designed to be $2 / 3$ theory and $1 / 3$ practical. The program employs teaching techniques including role-play and case-study approaches known to enhance practice change. Local faculty and international faculty will deliver the training curriculum.

\section{Patient and Provider Tools}

Provider and patient tools were translated and adapted for use in primary care settings in Greece. A tool kit of resources will be distributed to providers which includes: patient tobacco use survey, provider consult form, provider medication reference sheet, patient quit plan booklet, and waiting room posters. The provider consult form used a checklist style setup and provides real-time reminders for conducting an initial smoking cessation visit and follow-up appointments. The TiTAN Crete tools are available online at www.titan.uoc.gr

\section{Control Group}

Primary care providers in the control group will not be exposed to the intervention program.

\section{Procedures}

\section{Clinician recruitment}

An invitation letter will be sent to both intervention and control group practices explaining the objectives of the study and the study methods. A follow-up phone call/visit will be made to providers by a member of the investigative team one week after the invitation was sent to confirm interest in participation. An investigator will participate in one of the GP's monthly meetings to review the study protocol, consent forms and answer any questions. All GPs who agree to participate will complete an information sheet, consent form and a baseline survey. The provider survey will be repeated in intervention practices four months after the start of the program.

\section{Pre Intervention Data Collection}

Consecutive patients will be screened for eligibility in the waiting rooms of all participating GP offices. Screening for eligibility will be performed using a simple screening form. In order to be eligible to participate patients need to be: 18 years of age or older; current tobacco users ( $>5$ cigarette per day on most days of the week); seen in clinic for a nonurgent medical visit, and, able to read and understand Greek. Eligible patients who agree to participate in the study will provide informed consent and at the end of their clinic appointment will then be asked to complete the study survey. The survey will collect information about both outcome and predictor variables.

\section{Post-Intervention Data Collection}

The methods described in the pre-assessment will be repeated 4-months following the implementation of the intervention program to assess changes in the outcomes of interest. This will include repeating the provider survey and the data collection from a second cross-sectional sample of patients in all intervention practices.

\section{Outcome Measurement}

\section{Provider Knowledge, Attitudes, Subjective Norms}

Knowledge (6-items), attitudes (6-items), subjective norms (4-items), and perceived behavioral control (6-items), and intentions (6-items) regarding the treatment of tobacco use will be assessed by survey at the pre- and post-assessment with all providers. The survey instrument was developed based on previous research that examined the behavioral factors most closely associated with tobacco treatment delivery and uses a 5 -point Likert scale (strongly agree to strongly disagree) ${ }^{22,23}$.

\section{Barriers}

Provider's barriers to the delivery of tobacco treatment 
(10-items) will be assessed. A 4-point Likert scale will be used to rate the importance of each of the barriers to the providers' practice $(0=$ not at all important, $3=$ very important $)$. Barriers to be assessed include: disinterest in quitting; patients do not comply; lack of impact on patients; lack of time; lack of/ insufficient reimbursement; lack of patient education material; lack of training; lack of community resources to refer patients; complexity of smoking cessation guidelines; and, the perceived priority of other health problems.

\section{Provider Performance in the Delivery of Cessation Treatments}

Performance in the delivery of each of the 5As (ask, advice, assess, assist, arrange) will be assessed using an exit survey. The survey will instruct participants to respond either "yes", "no" or "don't know" when asked whether their provider asked them about their smoking status (ask); advised them to quit smoking (advise); assessed their readiness to quit (assess); provided assistance with quitting (assist); prescribed pharmacotherapy, provided self-help materials, and arranged follow-up support (arrange). Exit interviews or surveys have been used to assess 5 As delivery in several primary care trials ${ }^{11,22}$.

\section{Predictor Voriables}

Practice, provider and patient level variables will be assessed and are described here.

\section{Practice levelvariables}

Geographic location of clinic (postal code, rural/suburban); number of providers in practice, availability of allied health professional support.

\section{Provider level variables}

Socio-demographic (age, gender); number of years practicing medicine, Professional training (physician, nurse etc.), previous cessation training) will be assessed.

\section{Patient levelvariables}

Age, sex, ethnicity, years of formal education, occupation, income, and postal code will be documented. The presence of smoking-related illness will be documented using patient self-report including heart disease, stroke, chronic obstructive pulmonary disease, and cancer. The 4-item Patient Health Questionnaire (PHQ) is a diagnostic tool for mental health disorders used by health care professionals and can be completed quickly and easily ${ }^{24}$. The PHQ has been translated into Greek and validated for use ${ }^{25}$. The validation study among Greeks, found that overall the PHQ subscales demonstrated good psychometric properties, with the exception of the subscale examining problematic alcohol use. The two-item
Heaviness of Smoking Index (HSI) will be used to assess the degree of nicotine dependence. The HSI is a short form of the Fagerstrom Test of Nicotine Dependence. The HSI has been validated against other, 'gold-standard' tests of nicotine dependence ${ }^{26}$. The HSI score can range from 0 to 6 and is calculated by summing the number of cigarettes smoked daily and the time, in minutes, to first cigarette after waking. Higher HSI scores reflect greater tobacco use dependence. Smoking history will be assessed by documenting the number of years a participant has been smoking. The number of past quit attempts (lasting 24 hours or longer) in the past year will be documented).

\section{Theory of Planned Behaviour Constructs}

Patient attitudes ("How important is it for you to quit smoking?"), norms (Do others smoke in your home? Do most of your friends smoke? Do most of your colleagues at work smoke? How important is your physicians advise to quit smoking?), and perceived behavioural control (How confident are you that you can quit smoking?) will be assessed.

\section{Motivation to Stop Smoking}

The Motivation to Stop Smoking Scale will be used to assess a participant's motivation to quit ${ }^{27}$. This single item tool asks respondents: "Which of the following describes you?" Response options are: (1) "I don't want to stop smoking"; (2) "I think I should stop smoking but don't really want to"; (3) "I want to stop smoking but haven't thought about when"; (4) "I REALLY want to stop smoking but I don't know when I will"; (5) "I want to stop smoking and hope to soon"; (6) "I REALLY want to stop smoking and intend to in the next 3 months"; (7) "I REALLY want to stop smoking and intend to in the next month".

\section{Bio-statistical considerations Samplesize and power calculation}

Power calculations were based on rates of provider delivery of tobacco treatment 'advice'. Given the clustered design an inflation factor was used to enlarge the total sample size to account for loss in statistical power. Based on estimates generated from previous research we have estimated that the ICC will be $0.01^{11,20}$. We have estimated rates of provider 'advice' will be $45 \%$ in the Control Group, $60 \%$ in the Intervention Group. The control group rate was estimated based on a previous sampling of primary care practices in Greece $^{12}$. We have assumed the effect in the current project to be $15 \%$ based on rates achieved based on previous research ${ }^{11}$. The sample size calculation, based on 25 practices (15 
intervention practices and 10 control practices), indicates 28 patients per practice. All calculations were based on a twosided test, with $90 \%$ power, and an alpha level of 0.05 . We have increased sample size to account for possible loss to follow-up among providers. A total of 36 patients will be sampled at each practice or clinic. Measurement will occur at two timepoints in intervention practices (pre- and post-intervention). Measurement in the control practices will occur at only one time point. The total sample at the pre-assessment will be 900 (25 clinics x 36 patients/clinic). The patient sample at the post-assessment will be 540 patients ( 15 clinics $\mathrm{x} 36$ patients/ clinic).

\section{Primaryanalysis}

Clinic, provider and patient characteristics will be compared between groups using t-tests for continuous variables and Pearson chi-square tests for categorical variables. Multilevel modeling will be used to examine intervention effects controlling for provider and clinic level clustering. The effect of the intervention will be estimated using iterative generalized least squares method for binary data. To understand the patient- provider- and clinic-level factors associated with each outcome, separate multi-level logistic regression analyses will be performed using backward (Wald) stepwise selection to choose significant interaction terms at the $5 \%$ level after entering all the main effects in the model. Wald tests will be used to obtain p-values and odds ratios with 95\% CI will be used to summarize the effect estimates.

\section{DISCUSSION}

In Greece, there are very high rates of tobacco use and tobacco-related disease. Engaging primary care practitioners to support patients with smoking cessation is of critical importance. Insufficient rates of evidence-based smoking cessation treatment delivery have been documented in primary care practice settings and are thus important targets for quality improvement initiatives. Developing intervention programs that address well-known barriers may increase rates of cessation delivery in primary care settings especially. Such interventions may be of particular significance given in the austerity conditions affecting Greece. These barriers include a lack of provider knowledge, attitudes, and confidence regarding tobacco treatment delivery, the lack of time in busy primary care practices, lack of organizational supports, and patient resistance ${ }^{28}$. The TiTAN-Crete program has been designed to address important barriers to tobacco treatment delivery in primary care.

Adaptation to local context is critical to successful knowledge translation programs. In this project we have engaged local providers in the adaptation of validated, evidence-based model and will develop and support its rollout in daily clinical practice to increase uptake and efficacy. Baseline data collection activities for the TiTAN-Crete intervention program began in May 2015 and post-data collection activities will be completed in April 2016. This study will lead to a better understanding of how best to assist clinicians in Greece with enhancing the rates at which smoking cessation treatments are delivered to smokers. Lessons learned will be used to inform the further expansion of such interventions in Greece - and potentially other European primary care settings.

\section{REFERENCES}

1. Filippidis FT, Vardavas CI, Loukopoulou A, Behrakis P, Connolly GN, Tountas Y. Prevalence and determinants of tobacco use among adults in Greece: 4 year trends. Eur J Public Health 2013, 23(5):772-776. doi: 10.1093/eurpub/cks148

2. European Commission. Tobacco: Special Eurobarameter 332. 2010.

3. Faculty of the Harvard School of Public Health. The Greek tobacco epidemic. 2011.

4. WHO. WHO global report: Mortality attributable to tobacco. . 2012. ISBN:978 9241564434.

5. Young JM, Ward JE. Implementing guidelines for smoking cessation advice in Australian general practice: Opinions, current practices, readiness to change and perceived barriers. Fam Pract 2001, 18(1):14-20. doi: 10.1093/fampra/18.1.14

6. Gaziano TA, Galea G, Reddy KS. Scaling up interventions for chronic

disease prevention: The evidence. Lancet 2007, 370(9603):19391946. doi:10.1016/S0140-6736(07)61697-3

7. Kahn R, Robertson RM, Smith R, Eddy D. The impact of prevention on reducing the burden of cardiovascular disease. Diabetes Care 2008, 31(8): 1686-1696. doi: 10.2337/dc08-9022

8. McIvor A, Kayser J, Assaad JM, et al. Best practices for smoking cessation interventions in primary care. Can Respir J 2009, 16(4):129-134

9. Fiore MC, Jaén CR, Baker TB, et al. Treating tobacco use and dependence: 2008 update. Clinical practice guidelines, 2008.

10. European Smoking Cessation Guidelines, 2012.

11. Papadakis S, McDonald PW, Pipe AL, Letherdale ST, Reid RD, Brown KS. Effectiveness of telephone-based follow-up support delivered in combination with a multi-component smoking cessation intervention in family practice: A cluster-randomized trial. Prev Med 2013, 56(6):390-397. doi: 10.1016/j.ypmed.2013.02.018.

12. Kotsoni C, Antonakis N, Markaki A, Lionis C. Do patients with chronic obstructive pulmonary disease receive smoking cessation advice and interventions in rural crete? Report from a medical audit study. Aust J Rural Health 2008, 16(6):385-386. doi: 10.1111/j.1440-1584.2008.01024.x.

13. Brotons C, Björkelund C, Bulc M, Ciurana R, Godycki-Cwirko M, Jurgova E, Kloppe P, Lionis C, Mierzecki A, Pi eiro R, Pullerits L, Sammut MR, Sheehan M,Tataradze R, Thireos EA, Vuchak J, EUROPREV network. Prevention and health promotion in clinical practice: the views of general practitioners in Europe. Prev Med 2005, 40(5):595-601. doi:10.1016/j.ypmed.2004.07.020

14. Papadakis S, McDonald P, Mullen KA, Reid R, Skulsky K, Pipe A. 
Strategies to increase the delivery of smoking cessation treatments in primary care settings: A systematic review and meta-analysis. Prev Med 2010, 51(3-4):199-213. doi: 10.1016/j.ypmed.2010.06.007.

15. Vogt F, Hall S, Marteau TM. General practitioners' and family physicians' negative beliefs and attitudes towards discussing smoking cessation with patients: A systematic review. Addiction 2005, 100(10):1423-1431. doi: 10.1111/j.1360-0443.2005.01221.x

16. Gottlieb NH, Guo JL, Blozis SA, Huang PP. Individual and contextual factors related to family practice residents' assessment and counseling for tobacco cessation. J Am Board Fam Pract 2001,14(5):343-351.

17. Vaughn TE, Ward MM, Doebbeling BN, Uden-Holman T, Clarke WT, Woolson RF. Organizational and provider characteristics fostering smoking cessation practice guideline adherence: An empirical look. J Ambul Care Manage 2002, 25(2):17-31.

18. Reid RD, Mullen KA, Slovinec D'Angelo ME, et al. Smoking cessation for hospitalized smokers: An evaluation of the «ottawa model». Nicotine Tob Res 2010, 12(1):11-18. doi: 10.1093/ntr/ ntp165.

19. Reid RD, Pipe AL, Quinlan B. Promoting smoking cessation during hospitalization for coronary artery disease. Can J Cardiol 2006, 22(9):775-780.

20. Papadakis S, Cole AG, Reid RD, Coja M, Aitken D, Mullen KA, Gharib M, Pipe AL. Increasing rates of tobacco treatment delivery in primary care practice: Evaluation of the "Ottawa Model for Smoking Cessation". Annals of Family Medicine (in press).

21. Ajzen I. Theory of planned behaviour. Organizational Behavior and Human Decision Processes 1991,50(2):179-211. doi:10.1016/07495978(91)90020-T

22. Katz DA, Muehlenbruch DR, Brown RL, Fiore MC, Baker TB. AHRQ Smoking Cessation Guideline Study Group. Effectiveness of implementing the agency for healthcare research and quality smoking cessation clinical practice guideline: A randomized, controlled trial. J Natl Cancer Inst 2004, 96(8):594-603. doi: 10.1093/jnci/djh103

23. Pbert L, Adams A, Quirk M, Hebert JR, Ockene JK, Luippold RS. The patient exit interview as an assessment of physician-delivered smoking intervention: A validation study. Health Psychol 1999, 18(2):183-188. doi: 10.1037/0278-6133.18.2.183

24. Lowe B, Wahl I, Rose M, et al. A 4-item measure of depression and anxiety: Validation and standardization of the patient health questionnaire-4 (PHQ-4) in the general population. J Affect Disord 2010, 122(1-2):86-95. doi:10.1016/j.jad.2009.06.019

25. Karekla M, Pilipenko N, Feldman J. Patient health questionnaire: Greek language validation and subscale factor structure. Compr Psychiatry 2012, 53(8):1217-1226. doi: 10.1016/j. comppsych.2012.05.008.

26. Perez-Rios M, Santiago-Perez MI, Alonso B, Malvar A, Hervada X, de Leon J. Fagerstrom test for nicotine dependence vs heavy smoking index in a general population survey. BMC Public Health 2009, 9:493-2458-9-493. doi: 10.1186/1471-2458-9-493.

27. Kotz D, Brown J, West R. Predictive validity of the motivation to stop scale (MTSS): A single-item measure of motivation to stop smoking. Drug Alcohol Depend 2013, 128(1-2):15-19. doi: 10.1016/j. drugalcdep.2012.07.012.

28. Gagliardi AR, Alhabib S, members of the Guidelines International Network Implementation Working Group. Trends in guideline implementation: A scoping systematic review. Implement Sci 2015, 10(1):54-015-0247-8. doi: 10.1186/s13012-015-0247-8.

ACKNOWLEDGEMENT The authors would like to thank the staff and clinicians of the primary care clinics involved in this study for their participation

CONFLICT OF INTEREST

Pipe A. has received speaker and consulting fees from Pfizer, Johnson \& Johnson, and Amgen.

All the other authors have completed and submitted the ICMJE Form for Disclosure of Potential Conflicts of Interest and none were reported.

\section{FUNDING}

This work was supported by Global Bridges: Healthcare Alliance for Tobacco Dependence Treatment and Pfizer Independent Grants for Learning and Change (GB-13522581)

PROVENANCE AND PEER REVIEW

Not commissioned;

Externally peer reviewed 